\title{
Study on early warning system of rice disease based on IOT
}

\author{
WANG Guo-wei ${ }^{a}$, Sun $\mathrm{Yu}^{\mathrm{b}}$ and Niu Tai-yang ${ }^{\mathrm{c}}$ \\ College of Information and Technology,Jilin AgriculturalUniversity,Changchun130118,china \\ a 41422306@qq.com, b398223842@qq.com, ${ }^{\text {c } 443752009 @ q q . c o m ~}$
}

Keywords: Internet of things; data mining; Socket.

Abstract: In order to solve the problem of automatic detection of agricultural environment data, the function of real-time data acquisition and processing and the detection and warning of rice diseases is realized. The networking, ZigBee development and space fuzzy data mining technology and established networking rice disease early warning system based on, the realization of the rice growth environment data real-time acquisition, transmission, processing and rice diseases SMS warning function. The research result of this thesis has in Jiutai Meihekou and other areas, to achieve the timely warning of rice diseases, promoted the agricultural production in our province in the development of the information age, reached the rice disease effective prevention and increase the income of farmers.

\section{Introduction}

The Internet of Things are perceived information technology, network transmission technology and the rapid development of new technologies such as high degree of integration on the basis of a new kind of came into integrated information application technology in recent years, which is growing at an unprecedented rate for social, economic and human life fields, known as a new industrial revolution ${ }^{[1]}$. The date of birth of The Internet of Things, it is applied to the synchronization among industry and other aspects of agriculture. In particular, it has been greatly used in the conditions have the facility size modern intelligent agriculture. This is because the Internet of Things technology can establish a full range of intelligent automatic detection system on the agricultural environment, especially in the crop growth environment temperature, humidity, light, temperature, and other agricultural crops status information, automatic acquisition, detection and other functions ${ }^{[2-8]}$. Compared to other crops, greater domestic demand for rice, the country's rice planting area is great. Therefore, we consider the establishment of a set Study on early warning system of rice disease based on IOT, to achieve real-time acquisition of rice growth environment detection and data processing functions such warning. So as to achieve rice production, and promote the province's agricultural production aimed at the development of the information age.

\section{Research Content}

For major rice producing areas of Jilin major pest problem, based on data mining technology, GPS technology, wireless sensor technology, network data transmission technology, analysis of pests and diseases using conventional patterns and trends and real-time data acquisition and analysis, to realize the early warning model of rice diseases and pests. And establish relevant database, plant diseases and insect pests of rice in real-time, fast and accurate warning forecast, provide the corresponding integrated control measures. To achieve rice production digital management to provide technical support. The main content is divided into four parts:

(1) Establish data collection wireless sensor networks;

(2) To identify the occurrence of rice pests and diseases and environmental laws between the data, build predictive warning model;

(3) Server side software for the development of rice pest monitoring and early warning system;

(4) PC client software for the development of rice pest monitoring and early warning system based on GIS and Internet of things;

Innovation points: 
(1) This system combines GPS technology, sensor technology and wireless network technology to build wireless sensor networks, for achieving rice pests and diseases prediction in real-time early warning system of environmental information collection and storage.

(2) Utilization of WebGIS, GPS, data mining, expert systems and networking technology, developed based on WebGIS and The Internet of Things Jilin rice pest and disease monitoring and early warning systems.

(3)To integrate The Internet of Things, GIS and spatial fuzzy data mining technology to establish rice disease early warning model;

\section{System design}

The system is mainly used B / S mode is designed to achieve a multi-server, multi-client data sharing service model. The main module of the system include: data acquisition servers, data receiving and processing server, messaging server, PC client, data collection terminal. The main chart their systems shown in Fig. 1.

collecting data: Wireless sensor network monitoring system is mainly used for real-time monitoring of rice growing environmental information, the need to achieve acquisition, wireless transmission, gathering and processing the internal temperature of rice growth cycle a number of environmental information ${ }^{[9,10]}$. Combine rice growing and rice growing environmental information information system must meet the following measurements and scope of environmental information, and to ensure its reliability. Environmental information required for measurement and measurement range see the table 1:

Table 1 The range of parameters and system measurement environment

\begin{tabular}{lll}
\hline measurement items & measurement range & Measuring mode \\
\hline Air temperature & $0-80^{\circ} \mathrm{C}$ & Continuously measure \\
Air humidity & $0-100 \%$ & Continuously measure \\
Water temperature & $-30-80^{\circ} \mathrm{C}$ & Continuously measure \\
Light intensity & $1-65532 \mathrm{lux}$ & Continuously measure \\
\hline
\end{tabular}

And in practical environmental monitoring among, we often have a variety of sudden changes and additional requirements. At this point, we need the structure and number of nodes in the topology changes, more cases are increasing or decreasing the number of nodes is required, and this system should have this feature.

Server design: The server is mainly used for data receiving, distribution, processing, early warning and SMS sending and receiving, etc.. Receive and parse data from the GPRS wireless gateway through the Internet wireless gateway ${ }^{[11,12]}$. Then carry out the function of storage and operation to realize the early warning, then forward the data to the client. Finally, the early warning information is sent to the designated management personnel in order to achieve the purpose of real-time data acquisition, reception and early warning. To achieve the detection and early warning of major diseases and insect pests of rice, improve the yield of rice.

Data storage and real-time display: The main process of data storage module: server to open the Socket monitor function, the first, to establish a data transmission connection with the TCP/IP. Then, by the wireless network, the data observation nodes in rice fields will be aggregated to its designated GPRS gateway, and finally the unified will be sent to the specified IP address (IP address). To achieve the monitoring of the data sent to the data transmission gateway function. Finally, after processing the received data into the database, and displayed on the server side. So as to achieve the remote monitoring of rice growth environment related data. 


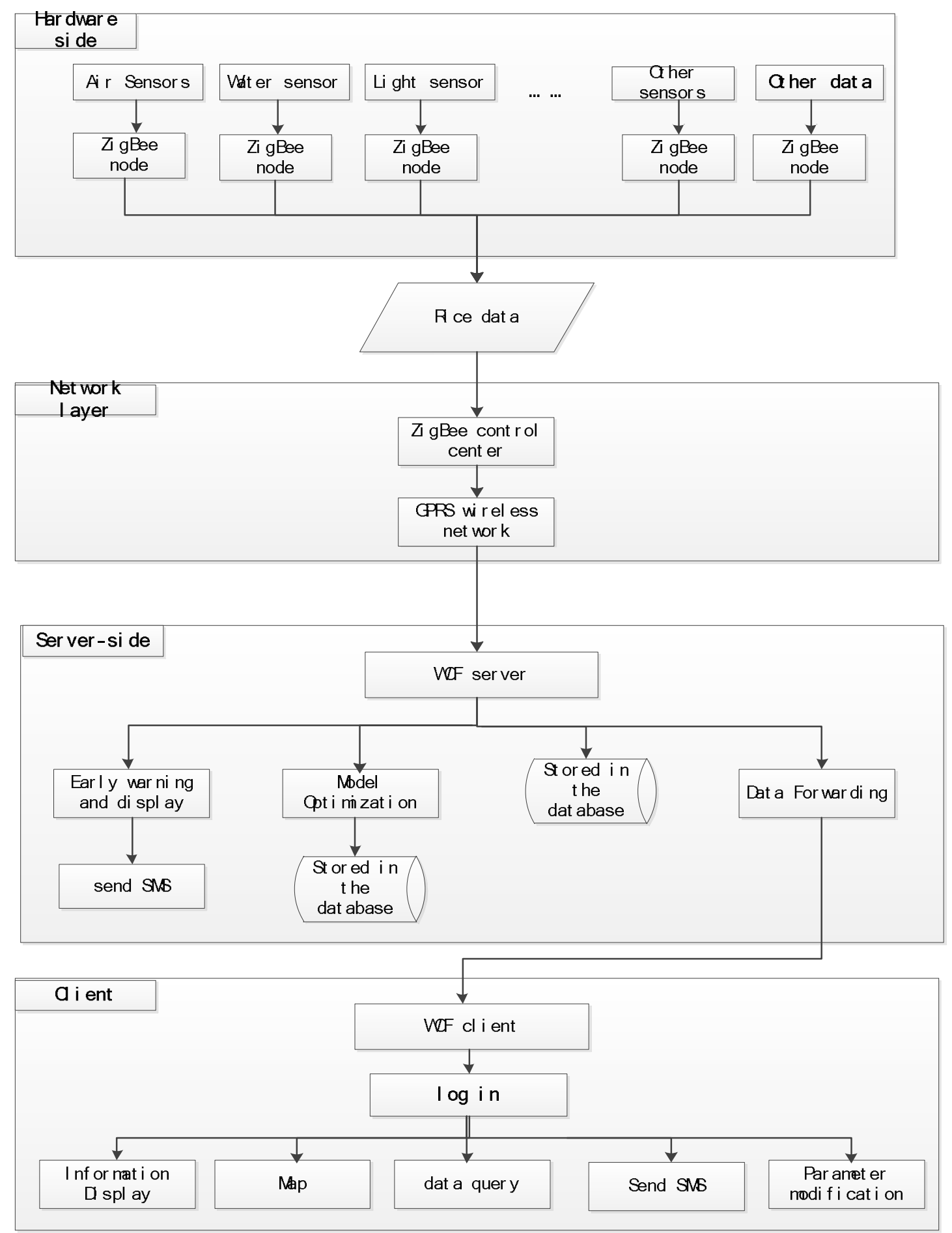

Fig. 1 System main architecture diagram

Data processing: Since the server from the Internet to receive data is byte array format, in the use of C\# processing, the use of NetworkStream and StreamReader class for data receiving and converting into String format, after the segmentation and recognition of the string, the data after the data classification into the storage.

Client design: The main function of the PC client comprising: receiving data sent by the server and parsing, historical data query, data of environmental parameters, real-time data to view, query warning message, bulk to farmers send early warning information ${ }^{[13,14]}$. Client system design is shown in Fig. 2: 


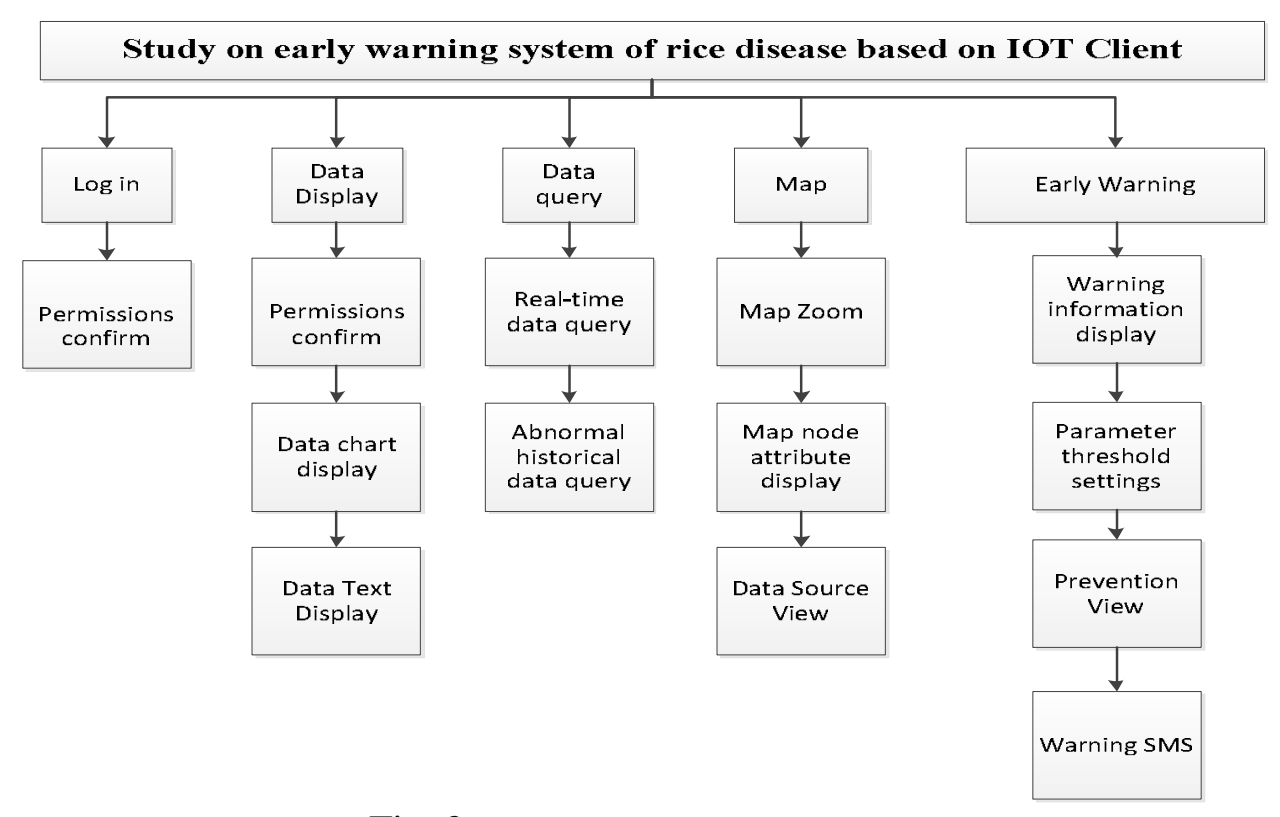

Fig. 2 The overall design of the client

Client Data Display: Data display is a PC client the most important functions, it provides users with instant rice growing environmental data and a variety of early warning information targeted to the user to remind the aim to reach the rice pest control. The system displays data in two modes, one is simple and clear text display, one is a more intuitive thermometer chart display mode. The first text display mode, the interface is in the information display area data sent by the server for direct display showing the current time period, location data is received, air data, temperature data and the light intensity data $^{[15-18]}$. The second chart shows the thermometer mode, the data sent by the server is converted to a digital format and then enter them into each chart display, dial into the air temperature, air humidity, dial, dial temperature and light intensity dial. At the same time, each will use double pointer mode dial, a pointer to the data display timely information, another pointer will display the value of the data in the normal state.

\section{Conclusion and future work}

In this paper, the use of GPS technology, sensor technology and wireless network technology, to build a rice pest and disease monitoring wireless sensor networks, real-time collection and storage of rice environmental information; the use of real-time data acquisition, historical data, the establishment of a rice disease prediction and warning related to database 3 ; fuzzy data mining technology, the establishment of a rice disease warning model; integrated WebGIS, GPS, data mining, expert systems and networking technology to build The Internet of Things based on WebGIS and Jilin rice disease monitoring and early warning systems.

The research results of this paper were identified as the international advanced level by the expert group of Jilin science and technology department. And in the Jilin Province Changchun City, Yushu City, Dehui City, Jiutai City, Meihekou city and Dongfeng County were application, effectively preventing the occurrence of rice diseases, the pesticide resources and improve the utilization rate of $20 \%$, disease prevention effect increases by $70 \%$, cumulative promotion area of 170 million mu, cumulative yield increase of 823 million $\mathrm{kg}$.

\section{Acknowledgements}

This work is supported by Jilin Province Science and Technology Department project (20150204058NY, 20140204045NY);Science and technology achievements transformation of agricultural science and technology(2014GB2B100001). 


\section{References}

[1] Dai Qiwei, Cao Jing, where Yan. [J]. design technology model of IOT oriented modern agriculture application of Jiangsu Journal of Agricultural Sciences, 2012 (5): 1173-1180., 28

[2] Sun Zhongfu, Ke Ming Du, Yin Shouyi. Development trend of Internet of things and agricultural applications prospect [J]. Agricultural information on the Internet. 2010, (5): 5-8.

[3] Chen Haiming, Cui Li, Xie Kaibin. Comparison of the structure and implementation of the Internet of things system [J]. computer, 2013,36 (1): 169-170.

[4] Qian Zhihong, Wang Yijun. Overview of wireless sensor networks for the Internet of things [J]. electronic and information technology, 2013,1:215-227.

[5] Su Mingyong, Liu Jitang. Research on the system of rice disease control and prevention [J]. the rural economy and technology, 2013, 9 (24):162-163.

[6] yuan Tao, Chen Xu, Ma super. Yangtze River farm rice diseases, insect pests and harmful integrated control system [J]. Chinese Agricultural Science Bulletin, 2013,29:182-186.

[7] Huang ting. Research on Key Technologies of the control node based on embedded [D]. 30-54. Zhejiang University, master's degree thesis:

[8] Chen Xu, Wang Tao, Tao Tao et al. Based on SOA to construct the integrated information service platform for rice plant diseases and insect pests [J]. Shanghai Agricultural Journal, 2012,28:127-131.

[9] Zhou Yinan, Zhou Yunsen. Application of wireless sensor network WSN in industrial production real-time monitoring [J]. industrial control computer, 2008 (8):24-25.

Cooper Joshua James, Anne.Challenges for Database Management in the Internet of Things[J].IETE Technical Review [10], 26, 2009 (5):320-329.

2008,4:86-88. [11] Yan Shang, Huang Zhang can.WCF_Windows windows platform is a new generation communication basic research and analysis $[\mathrm{J}]$. Computer and digital engineering.

[12] Zhou Yinglian, Liu Fu. Server load balancing technology [J]. computer and digital engineering, 2010,04:11-14.

Gomez J Huete, J F [13], O Hoyos, Interaction System based on Internet of Things as Support for Education [J]. Procedia Computer Science etal. 2013:132, - 139

Kwon S J. Conceptual modeling of causal map: Object oriented causal map[J]. Expert with Systems Applications [14], 38, 2011 (1): 360 - 370

[15] Wang Guowei, Yan Li, Chen Guifen. The comprehensive evaluation of the spatial difference of soil nutrients by variable rate [J]. Journal of agricultural engineering, 2009, 10:82-85.

Ng A Y Jordan, M I Weiss, Y. On Spectral Clustering: Analysis and an algorithm[J]. Advances in Neural Information Processing Systems [16], 2001:849-856.

Getz G. Coupled two-way clustering analysis of gene microarray data[J]. Proceedings of the National Academy of Science [17], 97, 2000 (22): 12079-12084.

[18] Wang Guowei, Yan Li, Chen Guifen. A weighted spatial fuzzy dynamic clustering algorithm [J]. computer engineering and applications, 2010,17:146-149. 